\title{
RESEÑA
}





\section{LOS CIENTÍFICOS Y EL MUNDO. LO QUE DIEZ PENSADORES NOS ENSEÑAN SOBRE LA AUTORIDAD DE LA CIENCIA Robert P. Crease (2020). Editorial Planeta.}

Robert P. Crease es estadounidense de nacimiento, filósofo y director del departamento de filosofía en la Stony Brook University en New York. Es autor de diversos libros relacionados al avance de la ciencia, por ello es considerado uno de los actuales historiadores de la ciencia. Desde siempre se ha preocupado por difundir el respeto y la autoridad de la ciencia, de tal forma que sea de utilidad para cada país. El estilo sensibilizador del autor en cada capítulo despierta el interés para terminar de leer todo el libro, asimismo se evidencia un profundo interés del autor por defender y demostrar la autoridad de la ciencia en todas sus areas.

El libro consta de 346 páginas divididas en cuatro secciones y que van precedidas por una dedicatoria y una introducción. La primera sección consta de tres capítulos y tiene el objetivo de mostrar la consolidación de la autoridad la ciencia en el inicio de la época moderna. La segunda parte también de tres capítulos muestra cómo es que durante los inicios de la ciencia, surgió un distanciamiento entre la ciencia misma y las humanidades y como es que se pudo salvar este distanciamiento. La tercera sección se enfoca en mostrar los primeros roces entre la política y la ciencia, es decir, cuáles fueron las primeras dificultades en el objetivo de que la ciencia pueda estar al servicio de la sociedad. Finalmente la cuarta sección está dedicado a Hanna Arendt, alemana y nacionalizada en Estados Unidos, filosofa y pensadora política, quien se ocupó de devolverle a la ciencia la autoridad que le corresponde a pesar de una evidente desconfianza de la clase política y de la misma sociedad.

El autor inicia en la introducción con una experiencia personal en su visita a la MER DE GLACE de Francia. Que era un atractivo turístico mundial, un extenso mar de hielo que ha servido incluso de inspiración para muchos escritores famosos como Goethe, Shelley entre otros. Sin embargo, para la visita del autor en el año 2018, se encontró que la MER DE GLACE cada vez es más pequeña y se sigue derritiendo como consecuencia de la emisión de dióxido de carbono a la atmosfera y que trae consigo el calentamiento global. De aquí se empieza a plantear el gran objetivo 
del libro: ¿si la ciencia está dando la información necesaria sobre las causas y consecuencias del calentamiento global, porque las autoridades políticas no toman acciones en base a informaciones científicas? La respuesta es clara: pues no le creen a la ciencia, y más bien consideran que la ciencia tiene sus propios intereses particulares. Según Crease, a esto se le llama el negacionismo de la ciencia. Es decir las autoridades políticas en el fondo pueden reconocer la autoridad de la ciencia, sin embargo, no les sirve para sus intereses políticos, por ello, una gran cantidad de autoridades políticas optan por el negacionismo de la ciencia. Justamente de aquí parte el Dr. Crease para desenmascarar al negacionismo de la ciencia, cada capítulo desde el primero hasta el décimo capítulo, tiene ese objetivo, restablecer la autoridad de la ciencia

El capítulo uno titula "la nueva Atlántida de Francis Bacon". El autor en un estilo ameno y sencillo va narrando lo que significó el aporte de Bacon, quien es considerado uno de los iniciadores de la ciencia moderna. Bacon nació en Londres, Inglaterra en el año 1561, en el seno de una familia de buenos recursos, su padre fue consejero de confianza de la Reina Isabel y su madre era pedagoga y traductora. Francis estudio y se graduó en derecho por deseo de su padre quien también lo quiso incursionar en la vida política de Inglaterra. De momento a otro el padre de Francis falleció y Bacon quedó en estado de pobreza pues no recibió ninguna herencia a diferencia de su hermano mayor; aún asi con esfuerzo personal y un poco de fortuna llego a ser diplomático como su padre, trabajando muy de cerca a la reina Isabel y luego de Jacobo I. En paralelo Bacon ya concebía sus ideas en torno a un trabajo sistemático con la naturaleza, a fin de sacarle el mayor provecho en beneficio de la sociedad; por ejemplo en aquellos tiempos las pandemias azotaban y cobraban muchas víctimas sobre todo en ni- ños. Francis Bacon escribió y publicó su famosa obra LA NUEVA ATLANTIDA, en donde a modo de parábola relata la historia de una embarcación que estando en el medio del mar, comenzó a sufrir dificultades como la falta de provisiones y además la aparición de enfermedades; cuando manera casual llegan a una isla misteriosa llamada Bensalem poblada por gente que se ha especializado en investigar todas las cosas con el objetivo de sacar el máximo provecho de ellas, como por ejemplo descubrir la cura para las enfermedades, etc. El mensaje que Francis Bacon intenta plasmar es que cada país debería establecerse talleres científicos con el objetivo de estudiar toda la naturaleza, es decir animales del mar, de la tierra y el aire, plantas, la tierra, etc, descubriendo sus propiedades $\mathrm{y}$ de sacarle provecho en beneficio de la sociedad, asimismo ejercer el control sobre la naturaleza. Este fue el mayor aporte de Bacon al mundo, fue el primero en proponer esta idea visionaria, y que con el pasar de los años se fue haciendo realidad en casi todo el mundo. Si bien es cierto Francis Bacon no fue científico, tampoco creó teorías, mucho menos fue inventor, algunos historiadores incluso afirman que Bacon fue un tipo materialista, interesado, ambicioso y hasta despiadado debido a algunas conductas poco altruistas que mostró a lo largo de su vida, sin embargo, su mérito radica en su deseo visionario de la implantación de talleres científicos para el bien de la humanidad. Bacon murió de neumonía a los 65 años de edad en el año 1626.

El capítulo II titula "Galileo Galilei y la autoridad de la ciencia". Galileo nació en Pisa, Italia en el año 1564. Galileo desde muy joven fue testigo de la implacable autoridad de la iglesia católica y por otro lado, el surgimiento del protestantismo religioso, asimismo de un nuevo movimiento que surgía lentamente pero a paso seguro: la autoridad de la ciencia. A diferencia de Francis Bacon, Galileo 
nació en un hogar humilde; otra diferencia es que Bacon con tal de lograr sus objetivos podía ser hasta condescendiente con sus enemigos, muy por el contrario Galileo no tenía reparos en responder abiertamente a sus adversarios con tal de defender sus convicciones. Desde muy joven se vio entusiasmado con las matemáticas, tanto asi que relacionaba los números con toda actividad cotidiana, como por ejemplo medir con su propio pulso el tiempo que tardaba la lámpara del techo en girar de un lado a otro, descubriendo que el tiempo de oscilación era el mismo ya sea en un arco pequeño o grande. Un detalle interesante es que a pesar que antiguamente se había realizado muchos avance en matemáticas, en la época de la predominancia de la autoridad religiosa, las matemáticas, no significaba nada interesante, por ello las ideas de Copérnico fueron atacadas por los teólogos de la época. En aquellos tiempos uno de los primeros enfrentamientos públicos de Galileo se dio con los filósofos de la época, quienes teóricamente proponían cómo funcionaba el mundo, sin embargo, no podían explicarlo con evidencias; ante esto Galileo realizó una publicación con cierto sarcasmo, afirmando que los filósofos no eran capaces de explicarlo, pues para ello, había que conocer de matemáticas. A partir del año 1609 empieza la construcción de lo que fue el primer telescopio creado con el objetivo de observar el espacio; llegando poco tiempo después a afirmar que agradecía a Dios por permitirle ver maravillas ocultas durante tantos siglos. A raíz de este descubrimiento trascendental, se pudo conocer más acerca de los cielos, de la luna, sin embargo, la declaración más revolucionaria fue confirmar la teoría de Copérnico: que la tierra y los demás planetas, giran alrededor del sol. Como otro dato curioso es que los filósofos de su época se negaron a comprobar por medio del telescopio las afirmaciones de Galileo. Las autoridades de la iglesia consideraron que dicho descubrimiento contradecía el texto de las sagradas escrituras: Eclesiastés 1:5. Galileo en un acto de querer manejar la situación sin querer provocar más a las autoridades religiosas, escribió una carta a la madre de una autoridad política: Cristina, en dicha carta Galileo argumenta que respeta a la iglesia, y que además tanto la ciencia como la biblia se complementan, es decir, si alguien rechazaba sus descubrimientos, en realidad no estaba comprendiendo la biblia, Dios había escrito dos libros: la biblia y el libro de la naturaleza. El argumento defensa no dio resultado, y el Papa Paulo V, ordenó una comisión de teólogos para que analicen el caso, en donde se concluyó que la teoría heliocéntrica era herética, sin embargo, debido a la influencia de algunos amigos, la iglesia no emitió ninguna sanción. En 1623, fue elegido Papa Urbano VIII, que era amigo suyo, a raíz de esto Galileo hizo una nueva publicación relacionado al Heliocentrismo, sin embargo, la publicación resulto ser muy provocativa, y al Papa Urbano VIII no le quedó otra opción que dejar el asunto en manos de la inquisición. El juicio se dio el año 1633, en donde Galileo se tuvo que retractar de la teoría del heliocentrismo y además lo condenaron a arresto domiciliario, refugiándose en su casa en las afueras de Florencia, en donde permaneció nueve años hasta el día de su muerte. Uno de sus más grandes aportes, aparte del telescopio, fue el ver a la naturaleza con el lenguaje de las matemáticas.

El tercer capítulo titula "Rene Descartes: el pensamiento del taller". Descartes nació 31 de marzo de 1596, al sur del valle Froila en Francia, prácticamente fue huérfano de infancia, pues su madre murió un año después de darle a luz, en un siguiente parto, y cuando Rene tenía cuatro años, su padre se volvió a comprometer; de tal forma que Rene fue criado por su abuela materna. Desde pequeño le gustó realizar sus actividades 
sin compañía de nadie, cuando estuvo en la escuela recibió el apodo de "don evasión". Estudió derecho por deseo de su padre, pero pronto se desaminó porque no le gustó que se discutía sobre palabras en lugar de crear cosas útiles. Se unió al ejército Holandés en donde se hizo amigo de Beeckman, que era un profesor visitante y aficionado a la ciencia. Luego de tal experiencia, Descartes se dedicó a viajar por diferentes lugares que le permitió conocer a destacados intelectuales de la época, teniendo el afán de encontrar un enfoque más sistemático para la ciencia de lo que habían propuesta Bacon y Galileo. De esta forma puso en práctica su propio procedimiento metódico. En la búsqueda de este método se inició disecando órganos de animales en su casa, lo cual le permitió conocer más sobre anatomía animal de lo que se conocía hasta esa época. Descartes llegó a concebir que tanto el sistema planetario como el organismo humano tienen funciones planetarias, es decir son como maquinas, uno de sus argumentos era que la tierra giraba alrededor del sol. Temía mucho por lo que pudiera pasarle, pues se había enterado de la situación de Galileo; ante esto, se encontraba en un dilema, pues Descartes respetaba mucho a la iglesia, pero además también precisaba de libertad intelectual para ser científico. En esas circunstancias publico EL DISCURSO DEL METODO, que por cierto es considerada su obra maestra. "Pienso luego existo", fue la primera verdad que plantea Descartes, afirmando que ante esta verdad cualquier creencia religiosa o moral seria irrelevante. Una cosa es pensar con el pensamento del taller y otra cosa pensar con el pensamiento ordinario de siempre. El mundo cotidiano de la vida y el taller científico pueden ser compatibles, es decir, uno dentro del otro. Es decir, descartes quería mejorar la forma de pensar de un creyente convencional por la de un experto metódico, la actividad numérica entonces ya no es solo de los científicos sino también de todos los habitantes, esta es la nueva forma de pensar que propuso Descartes.

El capítulo IV titula "Giambattista Vico: enloquecer racionalmente". Vico nació en Nápoles Italia el año 1668, siendo el sexto de ocho hermanos, en el seno de una familia pobre, en donde el padre trabajaba en una pequeña librería. Ya desde pequeño Vico mostró fortaleza de personalidad, sin imaginar las dificultades que la vida le deparaba. A la edad de siete años se cayó de una escalera de mano, llegándose a romper el cráneo; el médico que lo atendió pronosticó que el niño iba a morir en poco tiempo o en toda caso iba a vivir pero con retraso mental; pese a ello, Vico aun postrado, durante los siguientes tres años, se dedicó a leer todos los libros que pudo, tanto asi que a los 16 años ya pudo defender a su padre en un juicio legal. Por deseo de su padre estudio derecho, y según los historiadores están de acuerdo que en 1694 se licencio como abogado. Sin embargo, Vico no tenía el interés de ejercerlo, por ello, se ganaba la vida como profesor particular y paralelamente ya empezaba a escribir algunas cartas, poemas y otros textos. Su gran deseo era ser docente universitario, pero no lo conseguía debido a sus pocos contactos y su carácter solitario; finalmente en 1697 logro conseguir una plaza como profesor adjunto. El Gran aporte de Vico fue en favor de las ciencias humanas; Giambattista fue testigo en su época de dos formas de concebir el conocimiento: los métodos antiguos y los métodos modernos. Los defensores del método antiguo eran los relacionados a la filosofia, el lenguaje, las artes y por otro lado el método moderno se sustentaba en la abstracción, la demostración y la certeza, esparciéndose incluso el deseo deslegitimar al método antiguo, considerándola invalida y falsa. Giambattista Vico fue admirador de Francis Bacon, y por lo tanto también de las ciencias modernas, sin embargo, tuvo la 
genialidad de darse cuenta que no era saludable solamente educar a los niños con el método moderno de las ciencias, pues de esta forma se los convertía en personas con avaricia y egoísmo, dejando de lado su esencia humana, asimismo estaba de acuerdo que el método de la filosofia y de las artes, eran insuficientes por si solas, por lo tanto, propuso que los niños deben ser formados en ambas ciencias, es decir, primeramente desarrollar el lado metafórico, ingenioso y ambiguo del niño, y que de esta manera estaría preparado para desarrollar el pensamiento racional, abstracto y demostrativo que exigía la ciencia moderna, afirma que las ciencias naturales no pueden dejar de lado a la ética. Giambattista Vico murió en 1744. De esta forma Vico es considerado como uno de los grandes defensores de las humanidades sin rechazar el método moderno de la ciencia. Como bien menciona Robert Crease "el desastre fue la musa de Vico, la pobreza hizo de editora"; Vico no tuvo fortuna, tampoco reconocimiento en vida, incluso no aparece hoy en día entre los más conocidos pensadores, sin embargo, es digno reconocer su gran aporte a favor de las ciencias humanas.

El capítulo V, titula "la abominable idea de Mary Shelley". Mary Godwin Shelley Nació en Londres Inglaterra el año 1797. La vida de Shelley fue accidentada y complicada sobre todo desde su juventud hasta el día de su muerte en el año 1851. La obra literaria de Shelley ha trascendido siglos y generaciones hasta el día de hoy, e incluso ha sido tema central en diversas obras teatrales y películas en diversas versiones. Si bien es cierto Shelley publicó varias obras a lo largo de su vida, más se le recuerda por su obra cumbre Frankenstein. Pero ¿Cuál fue el motivo para que Robert Crease la considere en este libro de personalidades influyentes en el desarrollo de la ciencia? La respuesta es: la ética en investigación. Shelley tuvo la genial de idea de inmortalizar el personaje de Víctor Frankenstein, un científico apasionado, que quiso ser el pionero en descubrir poderes que la naturaleza ocultaba. Para ello empezó a diseccionar cadáveres por partes, y su intención era que por medio de un equipamiento eléctrico, generar vida en esos pedazos de seres humanos inertes; para ello se ocupó durante dos años, hasta que finalmente lo consiguió, logrando generar vida en aquel monstruo construido en base a pedazos de seres humanos. El Dr., Frankenstein quedo asustado con su creación e incluso se escondió, pero el monstruo comenzó a andar solo, convirtiéndose en un peligro, llegando incluso a asesinar al hermano del Dr. científico y finalmente llegando a asesinar a su propio creador: el Dr. Víctor Frankenstein. La historia de esta creación literaria de Shelley se hizo muy famosa, pero además lleva implícito un mensaje o una moraleja muy interesante: los científicos deben emplear la ética al desarrollar sus descubrimientos, de lo contrario se podrán crear nuevos monstruos que terminaran destruyendo a la misma humanidad que los creó. La pregunta obvia es ¿no será que actualmente existen nuevos Frankenstein en la ciencia? No solo en las ciencias naturales, sino también en las ciencias sociales o en las ciencias Biosociales. Por ello, la genialidad de Mary Shelley ha trascendido hasta la actualidad, porque en realidad se puede evidenciar que existen nuevos Frankenstein, por ello es importante el rol de la ética en la investigación, para que un grupo de expertos pueda evaluar la continuidad o no de una determinada investigación científica.

El capítulo VI titula "la religión de la humanidad de Auguste Comte". Este es un capitulo enfocado más en la personalidad del famoso filósofo francés Auguste Comte, más que en su propuesta positivista. Auguste Comte nació en Montpellier Francia, el año 1798. Ingreso a la escuela Politécnica de Paris, de donde fue 
expulsado por participar en un reclamo contra un profesor. Luego tuvo la oportunidad de conocer Henri Saint Simon que ya bordeaba los 70 años de edad, y quien sería influyente y determinante en el futuro académico de Comte. Existen evidencias bibliográficas para confirmar la personalidad Bipolar de Comte, presentando momentos de egocentrismo patológico y otras veces mostrándose inestable e inseguro; a raíz de su distanciamiento final con su maestro Saint Simon tuvo una crisis emocional entre los años 1826 y 1829, incluso se afirmó que paso una temporada en un hospital psiquiátrico y que tuvo dos intentos de suicidio; en estas circunstancias se casó con Caroline Massin, una mujer que se sabe que amó a Comte, sin embargo, Comte, al pasar de los meses la comenzó a tratar mal y humillarla. Otra característica notable era su narcisismo evidente, llegando a pensar en que era la persona indicada para salvar a la humanidad, otro detalle de su narcisismo era que no leía revistas ni periódicos de la época porque consideraba que no necesitaba hacerlo, pues estaba convencido que las mejores genialidades salían de su mente; en varias oportunidades despreció empleos para ser docente de instituto, porque consideraba que estaban por debajo del trabajo que el merecía. Comte, físicamente no tenía el aspecto de ser un caudillo o líder, siempre vestía con una levita negra (parecido a un saco) y un sombrero de copa, y quizá cualquier persona en la calle lo hubiera confundido como una persona inofensiva y hasta conservador. Era bajo de estatura, de contextura ancha, miope, una frente despejada y unas cejas rectas que resaltaban su mirada penetrante.

A Comte le pareció interesante las propuesta de Saint Simon, las cuales también el adoptó, como por ejemplo de que el mundo social estaba regido por leyes y que la ciencia sociales deben encontrarlas por medio de la investigación, de la misma forma que las ciencias naturales. Otro de los pensamientos que heredó de $\mathrm{Si}$ mon fue que la humanidad pasa por tres estados mentales en su evolución hacia la ciencia: el estadio teológico, el estadio metafísico o abstracto y el estadio positivo o científico. Consideró que los filósofos positivistas debieran actuar como los pastores religiosos, ayudando a que el rebaño de los seres humanos pueda llegar pronto a ese tercer estado: positivista o científico. Encontró en John Stuart Mill, filósofo inglés, un discípulo suyo, sin embargo, trato de aprovecharse solicitándole apoyo debido a sus peripecias económicas. Stuart Mill aceptó apoyarlo, pero Comte cada vez solicitaba más dinero, a esto se sumó las discrepancias académicas, lo que generó el fin de la amistad. Comte fundó el club positivista, que era como una nueva religión de la humanidad, en donde él sería el primer gran sacerdote; las reuniones se dieron cada semana con algunos seguidores fieles que tuvo. En la última etapa de su vida, adquirió y desarrolló un cáncer al estómago y finalmente murió el año 1857. Su gran aporte fue mostrar que la vida cotidiana y la ciencia pueden unirse y que a su vez estos van en constante cambio, y que hace falta hacer recordar a la humanidad el uso del enfoque experimental para superar las amenazas.

El capítulo VII titula "Max Weber: autoridad y burocracia". Max Weber nació en Alemania el año de 1864. El aporte de Weber se plasmó en sus famosas obras ECONOMÍA Y SOCIEDAD y LA ÉTICA PROTESTANTE Y EL CAPITALISMO, en donde se enfoca en proponer los diferentes tipos de autoridad que existían en la sociedad y su convivencia con la investigación. Un gran aporte visionario de Weber fue mencionar que si en una sociedad los políticos tuvieran mucha autoridad y los científicos muy poca autoridad, sería totalmente nefasto para dicha sociedad que iría inevitablemente hacia la deriva. Max Weber se licenció de abogado y además obtuvo un doctorado 
en economía, de hecho su tesis fue un gran aporte a la sociología. Solamente con 28 años, ya era un gran docente universitario y un gran abogado. Alto, con una gran barba y corpulento, con una gran debilidad por el alcohol, también ejerció como asesor del gobierno. A raíz de la muerte de su padre por un derrame cerebral cayó en un fuerte sentimiento de culpabilidad y una depresión severa, básicamente porque semanas previas Weber tuvo una fuerte discusión con su padre, lo que los distanció. Casi durante seis años Weber se retiró de sus labores académicas por causa de no superar la muerte de su padre; ya para el año 1903, la situación comenzó a mejorar. A lo largo de los siguientes años su interés estuvo relacionado a temas políticos, la burocracia, la ética y la ciencia, siendo una de sus convicciones que el gran problema de Alemania era el liderazgo político. Weber propuso tres tipos de liderazgo: la tradicional de los padres y ancianos, la racional legal que era la burocracia reflejada en normas y leyes de la sociedad y la carismática que es la que mayormente ejercen los líderes políticos; a su vez en ese liderazgo Weber detecta un conflicto: la ética de la responsabilidad y la ética de la convicción, de ahí que los intereses políticos están en constante antagonismo con la racionalidad de la ciencia. Los políticos defendiendo los valores del mundo y los científicos defendiendo los valores del taller, he ahí una explicación al negacionismo de la ciencia por parte de los políticos. A inicios del año 1919 le dieron la cátedra de economía y sociología en Múnich, estaba volviendo con fuerza a su intensa actividad académica, sin embargo, luego de contraer pulmonía, Weber falleció el 14 de junio de 1920. Como fruto de su esfuerzo intelectual, el economista y politólogo Max Weber hoy es considerado como uno de los padres de la Sociología.

El capítulo VIII titula "Kemal Atatürk: ciencia y patriotismo”. En este capítulo el autor se enfoca en la biografía de Mustafá Kemal Atatürk, quien fue el primer presidente de Turquía, es decir, no se trata de un científico sino más bien de un político, sin embargo, es una historia interesante que Robert Crease toma en cuenta en el desarrollo de la ciencia moderna. Kemal Atatürk nació en Turquía el año 1881, a la edad de 33 años se convirtió en el líder que derogó el gobierno islámico de su país; al ser proclamado como el primer presidente de Turquía, pronuncio un mensaje que causo polémica y dividió a la población turca; en dicho mensaje Atatürk mencionó que era necesario desarrollar la ciencia y el conocimiento para mejorar la civilización, para obtener una mejor calidad de vida y éxito. Aquel mensaje fue recibido con mucho fervor por un sector de la población, y con desagrado por el otro sector. Las convicciones religiosas islámicas invalidaban cualquier nuevo conocimiento que no sea del Corán; esta convicción estuvo arraigada durante siglos entre los clérigos religiosos y la población turca, lo cual impidió el desarrollo de su nación, porque no recibían nada que venga de Europa, a quienes consideraban seculares e infieles. Antes de Kemal Atatürk hubo intentos de copiar los avances modernos de Europa, pero estos intentos rápidamente fueron sofocados por presión de la iglesia islámica. Finalmente en 1924 Kemal Atatürk llega a ser presidente y desde el mismo comienzo está convencido de la utilidad de la ciencia y de sus beneficios para la población. Esta postura de su gobierno naturalmente generó nuevamente el rechazo de la iglesia islámica; el problema llegó a tal nivel que pronto Turquía se vio dividida en dos posturas: los que estaban a favor del conocimiento moderno y europeo y los que defendían a la sabiduría antigua mayormente turca y musulmana; incluso los líderes religiosos llevaron esta situación a un plano de patriotismo, de tal forma que todo aquel que aceptase 
el nuevo conocimiento de la ciencia estaba traicionando al sultán y además al imperio, es decir, si un ciudadano turco estaba interesado en aprender ciencia, era un antipatriota y traidor. Finalmente los defensores de la ciencia, de modo muy similar a lo que ocurrió en Europa, explicaron que en realidad la ciencia y el Corán no se contradicen, sino más bien una surge de la otra. De esta forma, poco a poco, los jóvenes fueron acercándose a la ciencia sin ningún sentimiento de culpabilidad. Luego de consolidar el avance de la ciencia en Turquía, Kemal Atatürk fallece en 1938; su liderazgo político, su mente visionaria, su espíritu guerrero y sus convicciones acerca de los beneficios de la ciencia, son méritos suficientes para que el Dr. Robert Crease lo haya considerado como una de las personalidades relevantes en el avance de la ciencia moderna en el mundo.

El capítulo IX titula "Edmund Husserl: crisis de la cultura". Husserl nació en Moravia el año 1859. Fue el segundo de cuatro hijos y además hijo de padres judíos, sin embargo, se convirtió al cristianismo y fue asiduo lector del nuevo testamento. Estudió y se doctoró en matemáticas, sin embargo, luego se especializó en filosofía. Fue docente universitario, asimismo fue testigo presencial del surgimiento del nazismo en Alemania y además víctima del antisemitismo, lo que expuso las actitudes poco leales de uno de sus más grandes discipulos: Martin Heidegger, quien al llegar a ser rector de la Universidad, y al ser simpatizante nazi, participó en la orden de separar a su maestro de la Universidad por ser de origen judío. Husserl consideraba que la filosofia podría convertirse en rigurosa igual que la ciencia analizando el modo en que la conciencia concibe al mundo. Previo a la concepción de fenomenología de Husserl, predominaba el naturalismo y el historicismo. El primero concebía al mundo y la realidad desde un taller científico, es decir comprobar teorías desde el taller y el segundo en contraste más bien considera que la realidad está fuera del taller, pues ahí fuera están los investigadores y los seres humanos. El naturalismo reduce la realidad del universo a un sistema causal, por otro lado, el historicismo concibe a los fenómenos desde diferentes perspectivas y que además pueden variar de una generación a otra. El problema con las ciencias naturales es que con su método científico quieren medir todo desde el punto de vista del objeto, sin considerar las emociones y fenómenos que experimenta el sujeto o una persona; en contraparte el problema con el historicismo es que separan un fenómeno del otro para estudiarlo. Husserl se enfrenta a estas dos posturas e inserta su propuesta de fenomenología, según Husserl el mundo en que vivimos es nuestra realidad cotidiana que a su vez está dentro del espacio y tiempo; explica además que existe un objeto real y un fenómeno, en donde el objeto real que existe por sí mismo independiente de que las conozcamos o no, y por otro lado, el objeto como fenómeno, aparece luego de haber sido conocido; asimismo una característica de todo fenómeno es el lugar que ocupa en el espacio la cual podemos percibir u observar desde diversos ángulos. Para ello, la certeza que puedo tener de un fenómeno es la de aquella que se aparece delante de nosotros tal y como esta, es decir, ya no son necesario los supuestos, a esto es lo que Husserl llamó el fenómeno puro, y entonces ese trance desde el objeto real al fenómeno puro se llama reducción fenomenológica. De esta forma planteó como podemos llegar a las cosas mismas para conocerlas, además de proponer un nuevo método para superar las limitaciones del naturalismo y el historicismo. Edmund Husserl murió el año 1938, y se sabe su discípulo Heidegger no fue a su funeral, quien tiempo después afirmó que estaba enfermo de gripe. El trabajo de Husserl despertó muchos seguidores y discipulos renombrados, por 
ello, hasta la actualidad su obra sigue siendo difundida.

El capítulo X titula "Hannah Arendt: Acción". Hanna nació en Alemania el año 1906, fue hija de inmigrantes judíos, llegando a convertirse en una pensadora influyente, además de ser una seguidora de Edmund Husserl y Martin Heidegger. Entre los episodios más resaltantes de su vida está el haber escapado del régimen nazi a primero a París y luego hacia Estados Unidos; por otro lado, algunas aventuras amorosas, incluso de manera secreta con Martin Heidegger. Los padres de Hanna le enseñaron desde niña a defenderse de las actitudes antisemitas, lo que le enseño a defenderse; sus padres siempre quisieron que pueda lograr una profesión para que pueda gozar de todos los derechos como alemana. Hanna Arendt comenzó a interesarse por las actitudes antisemitas en diferentes partes del mundo. Ya en establecida en EEUU tuvo la oportunidad de publicar algunos artículos entre ellos "Nosotros, los refugiados" en donde explicaba de forma magistral las vivencias y experiencias de los judíos refugiados y de los que no tienen patria en general. Luego público su primer libro LOS ORIGENES DEL TOTALITARISMO dividida en tres partes: Antisemitismo, Imperialismo y Totalitarismo, en donde se enfoca en analizar las consecuencias devastadoras del régimen nazi al final de la segunda guerra mundial. En este libro Hanna no solo describe los acontecimientos del régimen totalitario nazi sino que también ensaya el porqué del surgimiento de un régimen totalitario, como el nazi en Alemania y el Estalinista en Rusia. Este fue el libro que le da el reconocimiento intelectual en EEUU. Durante los siguientes años Arendt continuo publicando ensayos, interesándose cada vez más es temas políticos, y llegando a analizar el porqué del uso de la mentira entre los políticos, lo cual va muy relacionada al negacionismo de la ciencia, y además trata de explicar porque los políticos estarían dispuestos a rechazar la ciencia. Arendt expone cuatro razones del negacionismo: primero, porque es burocrático, lo cual puede generar actividades egoístas en perjuicio del país; segundo, porque los científicos se desenvuelven en un entorno y un lenguaje diferente al de los políticos, es decir, la ciencia no considera juicios de valor; tercero, porque la ciencia no es definitiva, siempre esta actualizándose; y cuarto porque se teme la acción de la ciencia sobre la naturaleza. Estas razones de Hanna explican por qué la ciencia puede ser vulnerable ante los ojos de los políticos que además incitan a la población a no creerle a la ciencia porque "sospechosamente" puedan tener sus propios intereses. Hanna Arendt falleció en el año 1975 luego de algunos problemas con el corazón. Haber tenido contacto con filósofos de renombre, recibir su influencia, asi como el desarrollo de su obra intelectual sobre el antisemitismo, los regímenes totalitarios y además las conductas de los políticos frente a los expertos o científicos, fue determinante para el merecido reconocimiento de su obra.

Rolly Guillermo Rivas Huaman Psicólogo

Grado de maestría. Cursando doctorado en Psicología 